\title{
Dynamic Fracture Experiment of Medium with Defects under Impact Loading
}

\author{
Chenglong Xiao $\mathbb{D}^{1},{ }^{1,2}$ Renshu Yang, ${ }^{1,3}$ Chenxi Ding, ${ }^{3}$ Cheng Chen, ${ }^{3}$ Yong Zhao, ${ }^{3}$ \\ Yiqiang Kang, ${ }^{1}$ and Guoli Shi, \\ ${ }^{1}$ State Key Laboratory for Geomechanics and Deep Underground Engineering, \\ China University of Mining and Technology (Beijing), Beijing 100083, China \\ ${ }^{2}$ School of Mechanics and Civil Engineering, China University of Mining and Technology (Beijing), Beijing 100083, China \\ ${ }^{3}$ Civil and Resource Engineering School, University of Science and Technology Beijing, Beijing 100083, China
}

Correspondence should be addressed to Chenglong Xiao; xcl_cumtb@163.com

Received 27 April 2020; Revised 19 July 2020; Accepted 1 September 2020; Published 16 February 2021

Academic Editor: Fabio Rizzo

Copyright (c) 2021 Chenglong Xiao et al. This is an open access article distributed under the Creative Commons Attribution License, which permits unrestricted use, distribution, and reproduction in any medium, provided the original work is properly cited.

\begin{abstract}
Defects have a significant effect on the dynamic fracture characteristics of the medium. In this paper, the dynamic fracture experiment of specimens with bias precracks is designed by utilizing the digital laser dynamic caustics system, and the effect of defect eccentricity on the dynamic fracture behavior is studied. Research shows that crack propagation can be divided into four stages: crack initiation stage, attraction stage, repulsion stage, and specimen fracture stage. The change of defect eccentricity has no obvious effect on the crack propagation behavior in the crack initiation stage and penetration stage but has a significant effect on the attraction stage and specimen fracture stage. In the process of the interaction between defect and crack, mode I stress intensity factor decreases at first and then increases. The decrease of mode I stress intensity factor reduces with the increase of defect eccentricity. The value of mode II stress intensity factor changes from negative to positive. With the increase of defect eccentricity, the symbol of mode II stress intensity factor no longer changes. The fractal dimension and the deflection angle of crack trajectory both decrease with the increase of defect eccentricity. In addition, a numerical simulation of the experiment is conducted by ABAQUS, which provides results that are in good agreement with the experimental results.
\end{abstract}

\section{Introduction}

The dynamic fracture of materials with defects is one of the principal topics in the fields of impact dynamics and fracture mechanics [1-3]. In many projects (building earthquake resistance, road and bridge engineering, rock roadway blasting engineering, etc.), engineering materials inevitably have defects such as microcracks and microholes. Affected by the above defects, dynamic fracture characteristics of materials change significantly, and the stability of the engineering structure is even affected [4-6]. Therefore, it is of great engineering significance to study the dynamic fracture behavior of materials with defects.

In recent years, many experts and scholars have conducted research on the dynamic fracture of medium with defects. In numerical simulation, Zhu et al. [3] simulated the effect of two empty holes with different spacing on running cracks propagation in sandstone and PMMA materials by AUTODYN. Yang et al. [7] analyzed the characteristics of stress distribution near void defects with different diameters in the process of crack propagation by extended finite element method (FEM). Haeri et al. [8] simulated the influence of the length and angle of precracks on the fracture path of Brazilian disk specimens by PFC2D. Rabczuk et al. $[9,10]$ described a new approach for treating crack growth by particle methods. This method performed quite well for rock damage and fracture problems. Ren et al. [11] analyzed the dynamic crack propagation by DH-PD, and research shows that the DH-PD can improve the stability of crack propagation.

In laboratory experiment, the caustics method provides an effective way to study the dynamic propagation behavior of cracks. This method is based on the principle of optical 
geometry, and the caustic image is utilized to characterize the complex problem of stress concentration at the crack tip. The corresponding dynamic fracture characteristic parameters can be obtained by analyzing the image [12]. Yang et al. [13] established a new digital laser dynamic caustics experimental system and applied the system to study the crack propagation behavior under the effect of the explosion or impact load. Yao et al. [14] used the dynamic caustics test method to study the stress intensity factor, propagation velocity, and propagation path of the crack of three-point bending specimens with offset precrack under impact loading. Yue et al. [15] studied the dynamic fracture behavior of mode I cracks at low loading rate and high loading rate based on the dynamic caustics experimental system. $\mathrm{Li}$ et al. [16] analyzed the influence of precrack angle on the crack propagation path and dynamic fracture characteristics of beam-column PMMA specimens.

Previous studies have greatly enriched the dynamic fracture theory of medium with defects and achieved many results. However, in practical engineering, there is not only the interaction between cracks and defects under symmetric structure but also the interaction under offset structure in more cases. Therefore, it is of more engineering significance to study the interaction between cracks and defects under offset structure. In this paper, a laboratory experiment combined with dynamic three-point bending and method of caustics is performed. The effects of defect eccentricity on the dynamic fracture behavior of PMMA specimens under the condition of bias precracks are studied. In addition, the fractal theory is applied to the analysis of crack growth trajectory, and the box-counting dimension is used as a parameter for quantitative evaluation of crack trajectory. Finally, the numerical simulation utilizing ABAQUS is conducted, which studies the change of stress field in the interaction range between crack and defect, so as to explain the cause of crack deflection and supplement the above experimental research.

\section{Dynamic Caustics Experiment}

2.1. Experimental System. In this experiment, the transmission digital laser dynamic caustics experimental system is applied. This experimental system consists of computer, FASTCAM SA5 (16G) high-speed camera, field lens, beam expander, laser device, drop hammer impact loading device, and so forth, as shown in Figure 1. Shooting frequency of the high-speed camera is $1.5 \times 105 \mathrm{fps}$ (the interval between two successive images is $6.67 \mu \mathrm{s}$ ), and the image size is $192 \times 168$ pixels. The output power of laser device is set to $50 \mathrm{~mW}$.

2.2. Experimental Principle. Caustics method is [17] based on the principle of geometric optics. The impact of the drop hammer causes cracks in the specimen. Under the action of tensile stress, the thicknesses of the materials near the crack tip change, which further influence the optical parameters such as refractive index. Light transmitted vertically through crack tip area is deflected and eventually forms caustics and caustic spot in the reference plane.
2.3. Experimental Design. The specimen material is polymethyl methacrylate (PMMA). The dynamic elasticity modulus and dynamic Poisson's ratio of PMMA are 6.1 GPa and 0.31 , respectively. The specimen is machined by laser cutting, as shown in Figure 2. The size of the specimen is $220 \mathrm{~mm} \times 50 \mathrm{~mm} \times 5 \mathrm{~mm}$. The precrack is processed at $6 \mathrm{~mm}$ to the right of the center of the lower boundary. The eccentricity $e$ of the elliptical defect in the center of the specimen is the only variable in this experiment. The semimajor axis $a$ of the ellipse is $3 \mathrm{~mm}$. The semiminor axis $b$ of schemes $S_{1} \sim S_{3}$ is $3 \mathrm{~mm}$ (round hole defect), $2 \mathrm{~mm}$, and $1 \mathrm{~mm}$, and the corresponding eccentricity is $0,0.75$, and 0.94 , respectively. In scheme $S_{4}$, the semishort axis is $0.3 \mathrm{~mm}$ and the eccentricity is 0.99 , which can be approximated as a crack defect. In order to verify the accuracy and repeatability of experimental results, there are 3 specimens in each scheme, and the experimental conditions are the same for each experiment. The experimental loading device is shown in Figure 3. The weight of the drop hammer is $1 \mathrm{~kg}$, and the falling height of the drop hammer in each experiment is $300 \mathrm{~mm}$.

Elliptical eccentricity is defined as

$$
e=\sqrt{1-\frac{b^{2}}{a^{2}}}
$$

where $e$ is eccentricity of ellipse; $a$ is the length of the semimajor axis; $b$ is the length of the semiminor axis.

\section{Cracks Propagation Path Analysis}

3.1. Fracture Morphology of Specimen. The results of three experiments in each group are basically the same. Each group randomly selected a specimen for analysis, named $S_{1-1} \sim S_{4-1}$, respectively. Figure 4 shows the fracture morphology of specimens $S_{1-1} \sim S_{4-1}$. For specimen $S_{1-1}$, the crack propagates toward the defect after initiation, and, under the influence of the defect, the propagation path deflects toward the defect but does not penetrate the defect. Then the crack propagation path gradually propagates away from the defect and propagates to the direction of the drop hammer loading. Finally, the specimen is penetrated by crack. According to the analysis of fracture morphology of above specimens, with the increase of defect eccentricity, the effect of defect on crack propagation decreases significantly.

3.2. Fractal Dimension of Cracks. Different from classical geometry theory, fractal geometry holds that the dimension of a figure can be defined as a fraction. The establishment of fractal theory provides a theoretical method for the quantitative representation of irregular geometric figures with statistical self-similarity [18]. Xie [19] introduced the fractal theory into rock damage and fracture analysis, studied rock damage and failure from the perspective of fractal geometry, and established a new way for rock damage analysis. Subsequently, many experts and scholars carried out further research. Peng et al. [20] studied the influence of image size on calculation accuracy in the calculation of fractal dimension for 2D digital images. Mao 


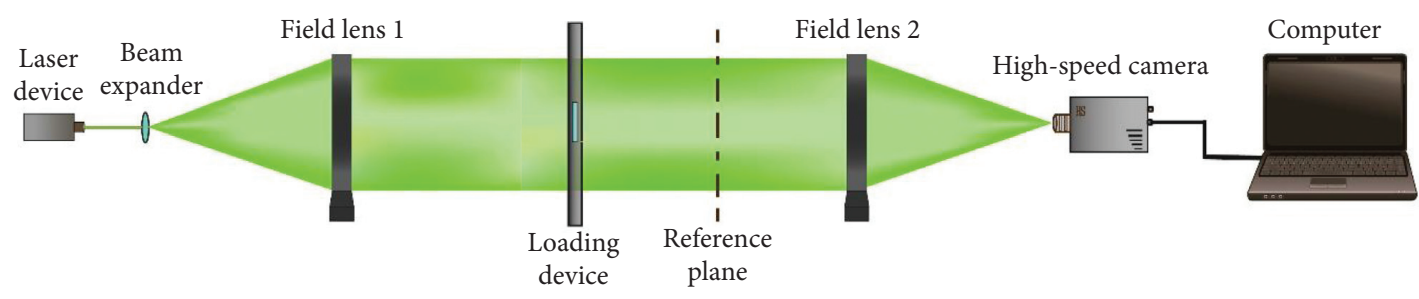

Figure 1: Dynamic caustics experiment system.

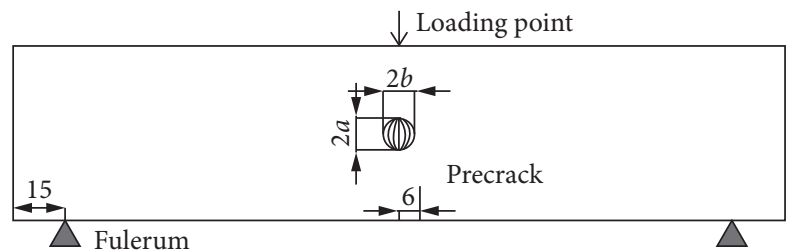

Figure 2: Schematic diagram of specimens.

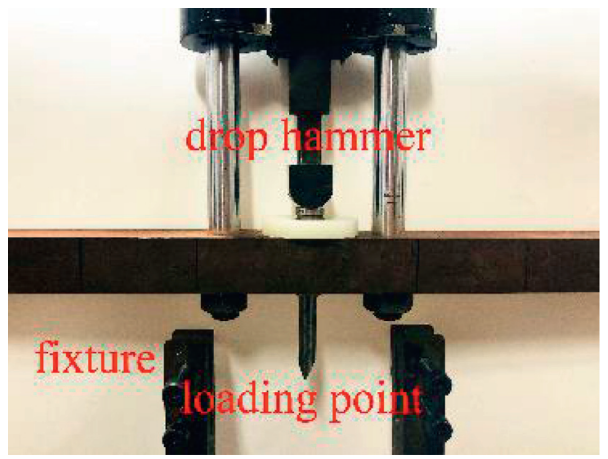

FIgURE 3: Impact loading device of drop hammer.

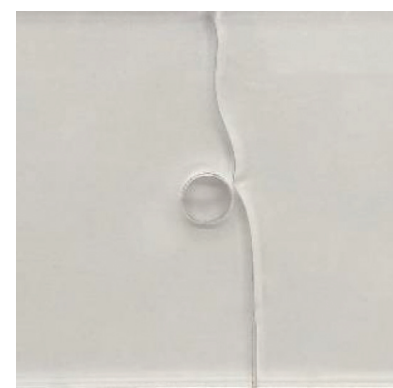

(a)

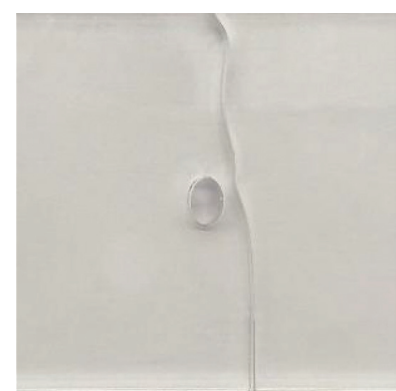

(b)

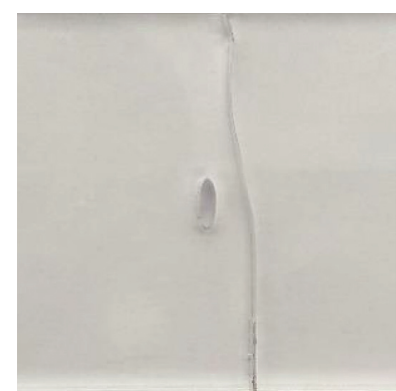

(c)

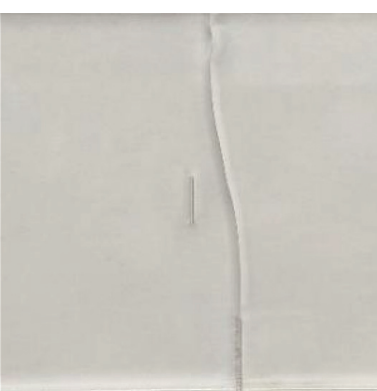

(d)

FIGURE 4: Fracture morphology of specimens. (a) $S_{1-1}$, (b) $S_{2-1}$, (c) $S_{3-1}$, and (d) $S_{4-1}$.

et al. [21] proposed a cube covering method to calculate the box-counting dimension for 3D digital images, and, based on this method, the mechanical properties of triaxial loading failure of coal samples were studied. Sun and Shangqu [22], based on the digital photogrammetry, counted the postexplosion profile of rock roadway and calculated its fractal dimension. It is considered that the fractal dimension can directly reflect the quality of roadway forming.
In this paper, the box-counting dimension is selected to analyze the crack propagation path. Schematic diagram of box division in box-counting dimension calculation is shown in Figure 5. Box-counting dimension [23] is defined as

$$
D_{B}=-\lim _{\delta \longrightarrow 0} \frac{\lg N(\delta)}{\lg \delta},
$$

where $\delta$ is the side length of the box and $N(\delta)$ is the number of boxes covering the fractal image. 


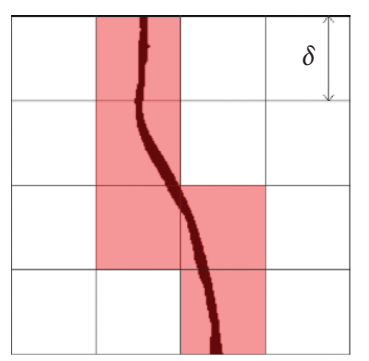

(a)

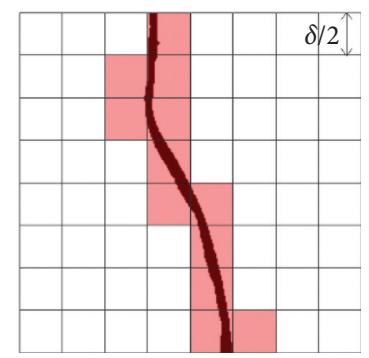

(b)

Figure 5: Box-counting dimension calculation. (a) The side of the box is $\delta$. (b) The side of the box is $\delta / 2$.

Catch the image of the crack propagation path in the $1 \mathrm{~cm}$ range near the defect. Import the image into MATLAB software for binarization, and obtain the binary image of the crack propagation path in this range, as shown in Figure 6. The size of the binary image is $256 \times 256$ pixels.

Import Figure 6 into MATLAB to calculate the fractal dimension of the crack propagation path. The fractal dimensions $D_{1} \sim D_{4}$ of the four cracks are $1.3100,1.2954,1.2634$, and 1.2546 , respectively. When the defect eccentricity is 0 , the fractal dimension of the crack trajectory reaches a maximum of 1.3100. Under this condition, the regularity of crack propagation path is the lowest, and the effect of defects on crack propagation is the most significant. When defect eccentricity increases from 0 to 0.75 , the fractal dimension decreases to 1.2954. When defect eccentricity increases to 0.94 , the fractal dimension decreases to 1.2634 . When defect eccentricity increases to 0.99 , that is, the defect is approximate to a crack, the fractal dimension reaches the minimum of 1.2546. Under this condition, the regularity of crack propagation path is the highest, and the effect of defects on crack propagation is the most insignificant. With the increase of defect eccentricity, the fractal dimension of cracks trajectory decreases, and the complexity of crack propagation path decreases, indicating that the influence of defect on moving crack weakens. The above analysis verifies the influence of defect eccentricity on nearby running crack from the view of fractal dimension.

3.3. Deflection Angle of Cracks. Figure 7 shows the relationship between crack deflection angle $\omega$ and vertical displacement $l$. The deflection angle $\omega$ at point $p$ is the angle between the tangent line and the vertical line at this point. The direction of the crack deflection to the defect is positive and the direction away from the defect is negative. It can be seen from the figure that the deflection angle stabilizes at about $5^{\circ}$ after crack initiation, and the deflection angle increases rapidly when the crack propagates near the defect. The maximum crack deflection angles of the four specimens are $29.4^{\circ}, 20.2^{\circ}, 17.8^{\circ}$, and $16.7^{\circ}$, respectively. With the increase of defect eccentricity, the maximum angle of crack deflection to defect decreases. When the crack propagates away from the defect, the repulsion phenomenon of the moving crack by the defects of $S_{1-1}$ and $S_{2-1}$ is significant, and the maximum deflection angles of the crack away from the defect are $10.6^{\circ}$ and $2.7^{\circ}$. With the increment of defect eccentricity $\left(S_{3-1}, S_{4-1}\right)$, the repulsive effect of defects on cracks decreases significantly. The above analysis verifies the influence of defect eccentricity on nearby running crack from the view of crack deflection angle.

\section{Time History Characteristic Analysis}

Dynamic caustic pictures of $S_{2-1}$ and $S_{4-1}$ at different times are shown in Figure 8. The impact time of the drop hammer on the specimen is recorded as $0 \mu \mathrm{s}$. As can be seen from Figure 8(a), showing the stress concentration at the tip of precrack after drop hammer impact on specimen, the energy gradually accumulates, and the caustic spot size enlarges. The caustic spot moves to the tip of precrack at $186.76 \mu \mathrm{s}$, and the specimen cracks. After crack initiation, the caustic spot extends to the defect and extends near the defect at $226.78 \mu \mathrm{s}$. The stress balance at the crack tip is broken by the attraction of the defect. The shape of the caustic spot changes, the size decreases, and it deflects toward the defect. The shape of caustic spot shows typical compound characteristics. After $280.14 \mu \mathrm{s}$, the size of caustic spot enlarges gradually and the caustic spot moves away from the defect. Subsequently, the size of the caustic spot decreases until $493.58 \mu$ s at which crack penetrates the specimen.

As can be seen from Figure 8(b), the size of caustic spot enlarges at the tip of precrack after drop hammer impact on specimen. The specimen cracks at $193.43 \mu$ s. After crack initiation, the caustic spot extends to the defect and extends near the defect at $246.79 \mu$ s. Due to the attraction of the defect, the caustic spot deflects toward the defect, the shape of the caustic spot changes, and the size decreases, but the range of change is much less than that of specimen $S_{2-1}$. After $286.81 \mu$ s, the size of caustic spot enlarges gradually and the caustic spot moves away from the defect. Subsequently, the size of the caustic spot decreases until $486.91 \mu \mathrm{s}$, at which crack penetrates the specimen.

\section{Stress Characteristic Analysis}

5.1. Stress Intensity Factor Analysis. The crack propagation path and deflection angle are affected by the stress field at the crack tip; that is, they are related to the release of energy. Measure the relevant feature size of the caustic spot in Figure 8 , and the dynamic stress intensity factor at the crack tip can be calculated from the following formulas $[24,25]$ : 


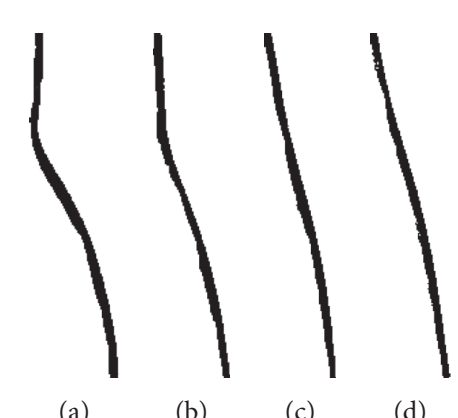

FIGURE 6: Binary image of crack propagation path. (a) $S_{1-1}$, (b) $S_{2-1}$, (c) $S_{3-1}$, and (d) $S_{4-1}$.

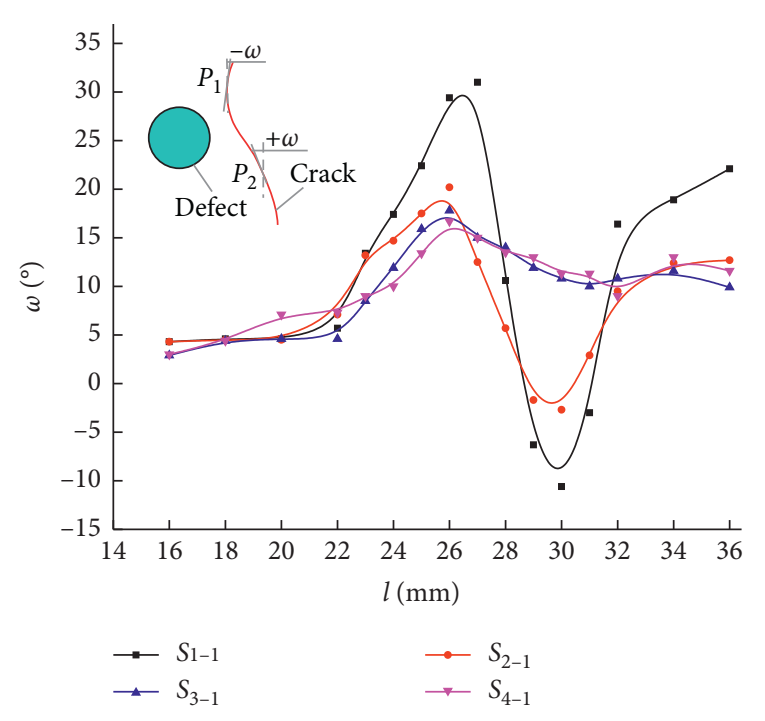

Figure 7: Curves of crack deflection angle with vertical displacement.

$$
\begin{gathered}
K_{\mathrm{I}}^{d}=\frac{2 \sqrt{2 \pi F(v)}}{3 z_{0} d_{\mathrm{eff}} c g^{5 / 2}} D_{\max }^{5 / 2}, \\
K_{\mathrm{II}}^{d}=\mu K_{\mathrm{I}}^{d}, \\
\mu=\operatorname{tg} \frac{\varphi}{2},
\end{gathered}
$$

where $K_{\mathrm{I}}^{d}$ is mode I stress intensity factor; $K_{\mathrm{II}}^{d}$ is mode II stress intensity factor; $z_{0}$ is the distance between the specimen and the reference plane, and, in this experiment, $z_{0}=0.9 \mathrm{~m} ; d_{\mathrm{eff}}$ is the effective thickness of the specimen, and, in this experiment, $d_{\mathrm{eff}}=5 \mathrm{~mm}$; is the optical stress constant of the material, and, for PMMA, $c=0.85 \times 10^{-10} \mathrm{~m}^{2} / \mathrm{N} ; D_{\max }$ is the maximum size of the caustic spot; $g$ is the numerical factor, and, in this experiment, $g=3.17 ; F(v)$ is the regulating factor of dynamic load, and, in practice, $F(v)=1 . \mu$ is the proportional coefficient of stress intensity factor, which can be determined by the angle $\varphi$ between the symmetry axis of caustics and the crack axis.

Figure 9 shows the time-varying curves of mode I stress intensity factor $K_{\mathrm{I}}^{d}$ during the crack propagation of four groups of specimens. Take the experimental curve of defect eccentricity 0.75 (specimen $S_{2-1}$ ) as an example. After the drop hammer impact on the specimen, the stress is concentrated at the tip of the precrack, and $K_{\mathrm{I}}^{d}$ increases accordingly. When $K_{\mathrm{I}}^{d}$ increases to $1.01 \mathrm{MN} / \mathrm{m}^{3 / 2}(180.09 \mu \mathrm{s})$, the specimen cracks, the energy at the slit is released after cracking, and $K_{\mathrm{I}}^{d}$ decreases rapidly. Affected by the defect, the crack deflects to the defect, and $K_{\mathrm{I}}^{d}$ continues to decrease and decreases to the valley $0.54 \mathrm{MN} / \mathrm{m}^{3 / 2}$ at $273.47 \mu \mathrm{s}$. Then the crack moves away from the defect, and $K_{\mathrm{I}}^{d}$ increases accordingly and increases to the peak $0.86 \mathrm{MN} / \mathrm{m}^{3 / 2}$ at $326.83 \mu \mathrm{s}$. After that, $K_{\mathrm{I}}^{d}$ decreases gradually until the specimen is penetrated by the crack. From the above analysis, the defects play a significant role in inhibiting crack propagation.

In this experiment, the evolution of mode I stress intensity factor $K_{\mathrm{I}}^{d}$ can be divided into four stages. The first stage is crack initiation stage: after the drop hammer impact on the specimen, the energy gradually accumulates at the tip of the precrack, and the specimen begins to crack when $K_{\mathrm{I}}^{d}$ increases to the critical value. Critical $K_{\mathrm{I}}^{d}$ of crack initiation of the four specimens are in the range of 0.95-1.05. The second stage is attraction stage of defects to cracks: after crack initiation, affected by the defect, the crack deflects toward the defect, and $K_{\mathrm{I}}^{d}$ gradually decreases to the valley. Compared with the crack propagation to the defect, $K_{\mathrm{I}}^{d}$ of the four groups of specimens decreased by $0.51,0.37,0.27$, and $0.15 \mathrm{MN} / \mathrm{m}^{3 / 2}$, respectively. With the increment of defect eccentricity, the decrease of $K_{\mathrm{I}}^{d}$ at crack tip reduces. From the changing law of stress intensity factor, it can be seen that the inhibitory effect of defects on running cracks decreases with the increase of eccentricity. The third stage is repulsion stage of defects to cracks: when $K_{\mathrm{I}}^{d}$ at the crack tip decreases to the valley, the crack propagates away from the defect, and $K_{\mathrm{I}}^{d}$ tends to increase. With the increment of defect eccentricity, the promoting effect of defect on $K_{\mathrm{I}}^{d}$ increases. The fourth stage is specimen fracture stage: after the crack propagates away from the defect, it propagates to the direction of the drop hammer loading, and $K_{\mathrm{I}}^{d}$ decreases until the specimen ruptures.

In order to further analyze the stress when the crack propagates near the defect, calculate mode II stress intensity factor $K_{\mathrm{II}}^{d}$ of the crack tip in the attraction and repulsion stages. Figure 10 shows a schematic diagram of the forces acting on a plane crack under different deflection conditions. From formula (4) and Figure 10, it can be seen that when the 


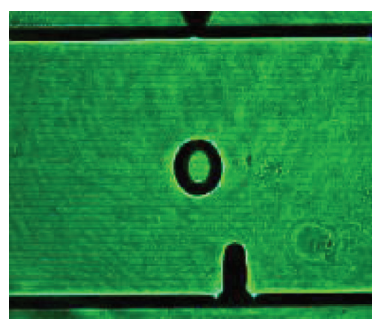

$t=0 \mu \mathrm{s}$

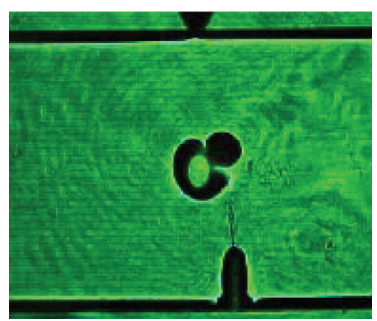

$t=280.14 \mu \mathrm{s}$

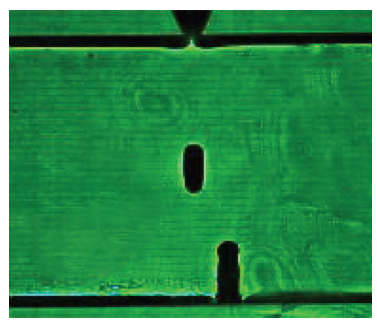

$t=0 \mu \mathrm{s}$

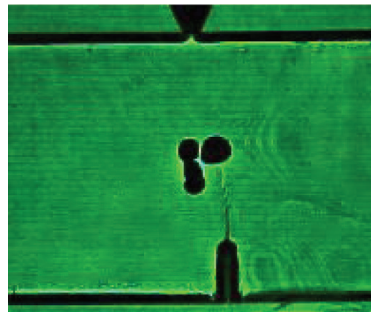

$t=286.81 \mu \mathrm{s}$

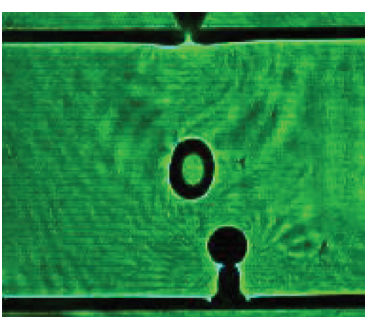

$t=186.76 \mu \mathrm{s}$

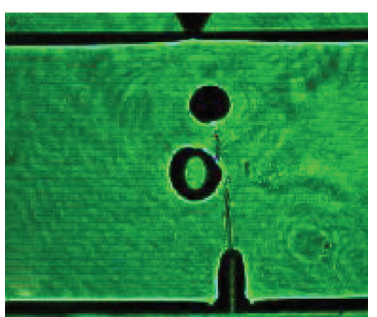

$t=320.16 \mu \mathrm{s}$

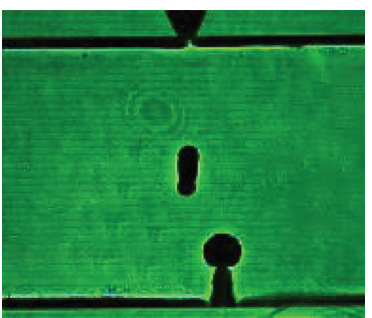

$t=193.43 \mu \mathrm{s}$

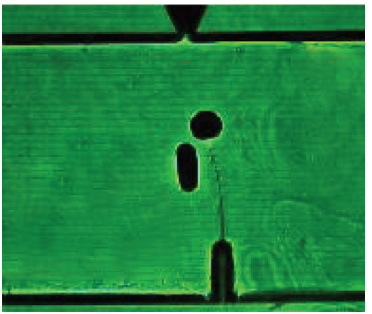

$t=313.49 \mu \mathrm{s}$

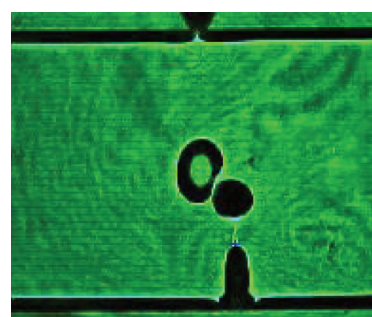

$t=226.78 \mu \mathrm{s}$

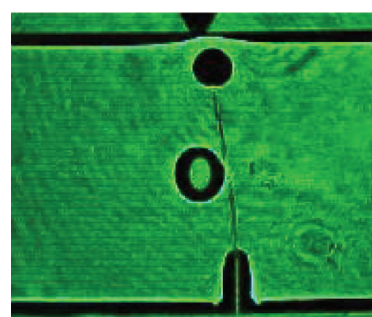

$t=366.85 \mu \mathrm{s}$

(a)

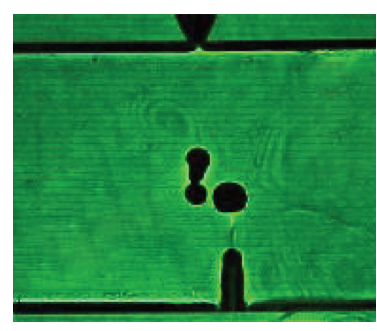

$t=246.79 \mu \mathrm{s}$

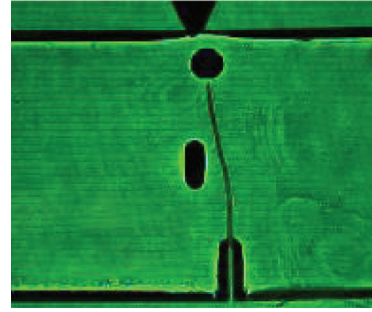

$t=360.18 \mu \mathrm{s}$

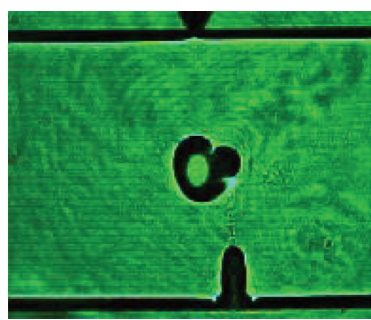

$t=260.13 \mu \mathrm{s}$

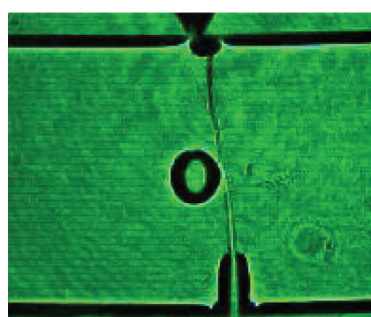

$t=493.58 \mu \mathrm{s}$

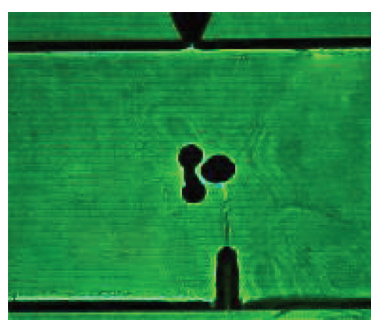

$t=273.47 \mu \mathrm{s}$

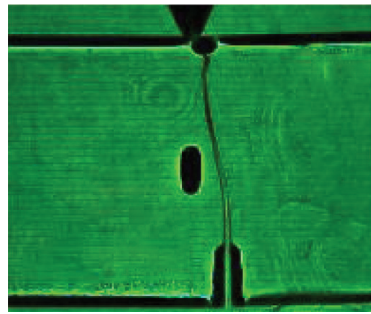

$t=486.91 \mu \mathrm{s}$

(b)

Figure 8: Dynamic caustic spots of specimens. (a) Specimen $S_{2-1}$. (b) Specimen $S_{4-1}$.

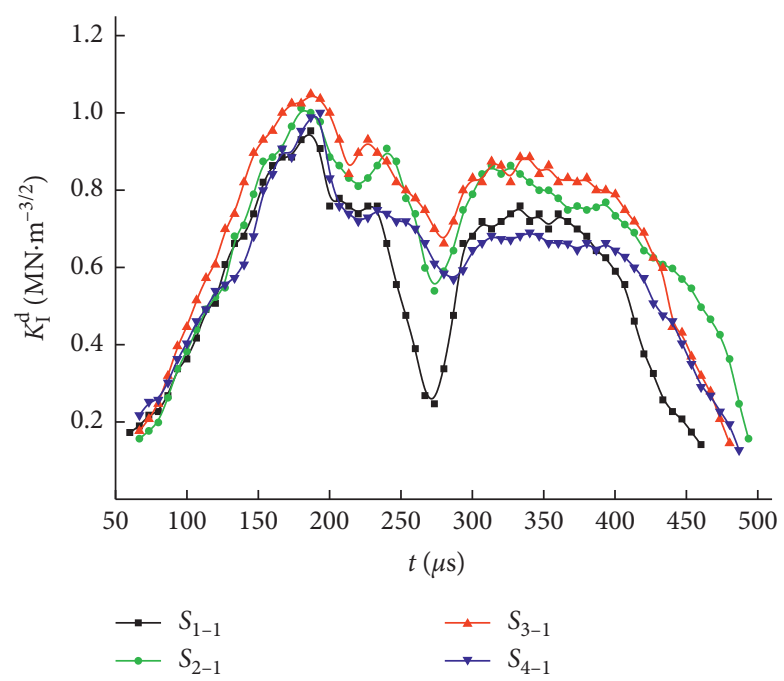

FIGURE 9: Curves of mode I stress intensity factor with time. 


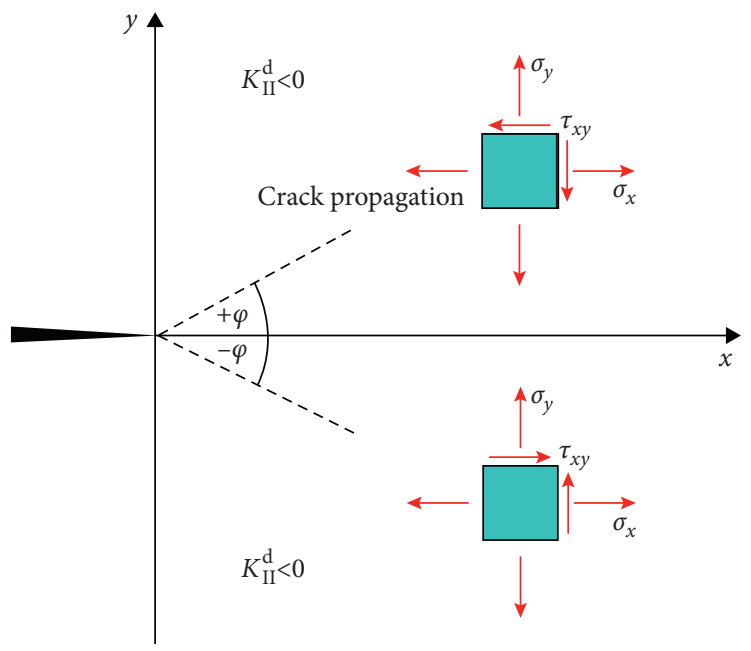

Figure 10: Schematic diagram of stress under different deflection of plane crack.

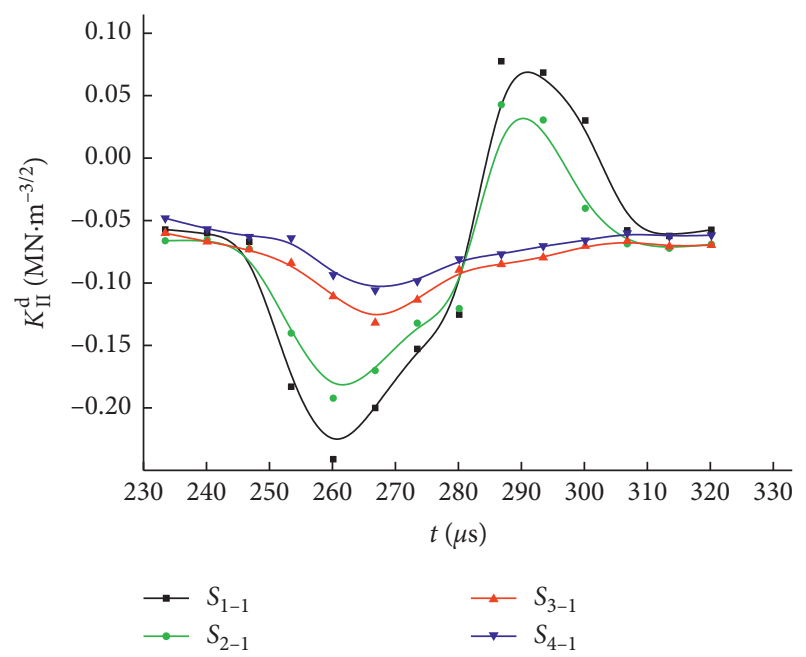

FIgure 11: Mode II stress intensity factor curves with time.

shear stress component $\tau_{x y}$ of the crack is positive, $K_{\mathrm{II}}^{d}$ and deflection angle are negative; when the shear stress component $\tau_{x y}$ of the crack is negative, $K_{\mathrm{II}}^{d}$ and deflection angle are positive.

Figure 11 shows the time-varying curves of mode II stress intensity factor $K_{\mathrm{II}}^{d}$ when the crack propagates near the defect. As can be seen from this figure, the $K_{\text {II }}^{d}$ valleys of the four specimens are- $0.24,-0.19,-0.13$, and $-0.11 \mathrm{MN} / \mathrm{m}^{3 / 2}$, respectively. The absolute value of $K_{\mathrm{II}}^{d}$ decreases with the increase of defect eccentricity. In the process of the interaction between cracks and defects, $K_{\text {II }}^{d}$ of $S_{1-1}$ and $S_{2-1}$ changed from negative to positive, and $K_{\mathrm{II}}^{d}$ of $S_{3-1}$ and $S_{4-1}$ remain negative. From the $K_{\mathrm{II}}^{d}$ variation law of $S_{1-1}$ and $S_{2-1}$, it can be seen that the direction of the shear stress component $\tau_{x y}$ at the crack tip changes from positive to negative. As the defect eccentricity continues to increase, the direction of $\tau_{x y}$ at the crack tip remains positive. The above analysis explains the change of crack propagation path and deflection angle in the process of crack-defect interaction from the view of force.
5.2. Numerical Simulation Analysis. In order to explore the influence of defects on the stress field at the crack tip in the process of crack propagation, ABAQUS is used to simulate this experiment. The extended finite element method (XFEM) is selected as the simulation method. The advantage of XFEM is that the mesh is independent of the geometric or physical interface of the specimen, thus overcoming the difficulties caused by high-density mesh generation in areas with high stress and deformation concentration, such as crack tip.

Because the parameters such as crack growth rate and stress intensity factor have been accurately measured in the dynamic caustics test, the simulation in this paper focuses on the change of stress field in the interaction between crack and defect in the process of crack propagation. By analyzing the stress concentration range of the specimen, the cause of crack deflection is studied, so as to supplement the above experimental research. In this numerical simulation, relevant parameters of PMMA are as follows: density of $1.18 \mathrm{Kg} / \mathrm{m}^{3}$, Poisson's ratio of 0.31 , and elasticity modulus of $6.1 \mathrm{GN} / \mathrm{m}^{2}$. The material of the specimen is simplified as an elastic material, and the damage model of the material is defined as the Maxps damage model.

Figure 12 shows the von Mises stress field of $S_{2-1}$ and $S_{4-}$ 1 with different vertical distance of the crack. As can be seen from the figure, the fracture form and propagation path of the crack are in good agreement with the experimental results. Because the precrack offsets from the center of the specimen by $6 \mathrm{~mm}$, the stress field at the crack tip is asymmetrical after the specimen cracks. With the propagation of the crack, the stress concentration appears in the upper and lower regions of the defect. The stress concentration range of specimen $S_{2-1}$ is larger than that of specimen $S_{4-1}$. Affected by the defect, the crack propagation path deflects toward the defect, and the stress concentration range increases accordingly. Comparing the stress clouds images of $S_{2-1}$ and $S_{4-1}$, it can be seen that the stress concentration range decreases with the increase of defect eccentricity. At $a=15.1 \mathrm{~mm}$, the crack deflection angle of specimen $S_{2-1}$ to the defect reaches the maximum, and then 

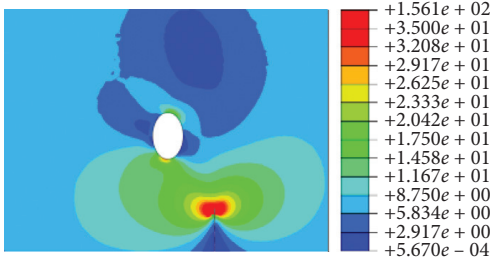

$a=0 \mathrm{~mm}$

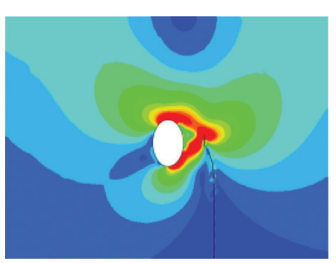

$a=15.1 \mathrm{~mm}$

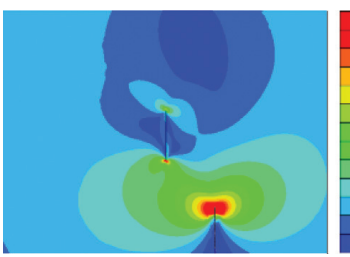

$a=0 \mathrm{~mm}$

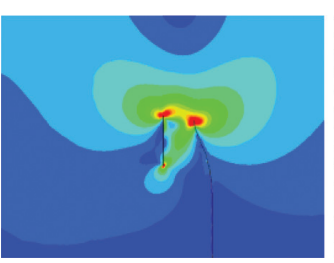

$a=15.4 \mathrm{~mm}$

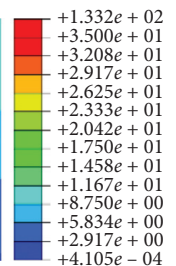

$.917 e+00$
$4.105 e-04$
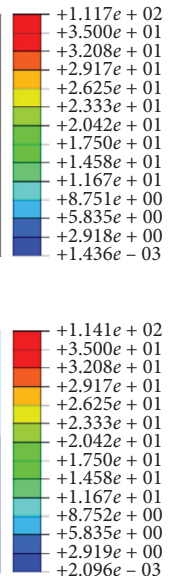

$2.919 e+00$
$+2.096 e-03$

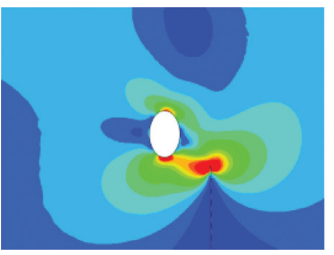

$a=6.2 \mathrm{~mm}$

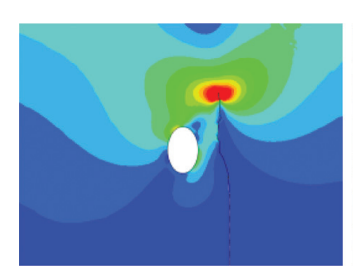

$a=16.4 \mathrm{~mm}$

(a)

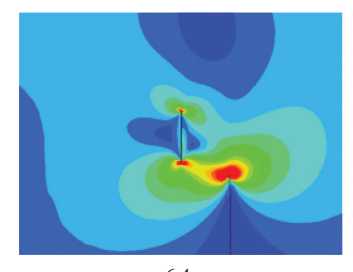

$a=6.4 \mathrm{~mm}$

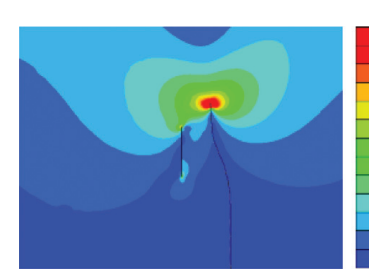

$a=17.4 \mathrm{~mm}$
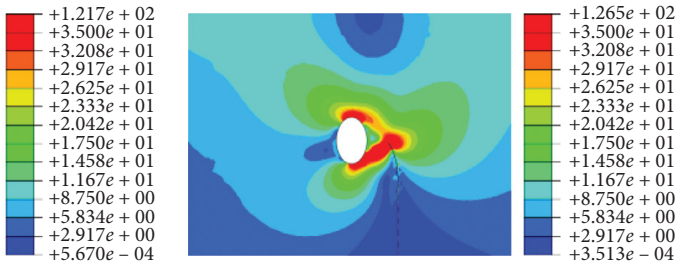

$a=11.4 \mathrm{~mm}$
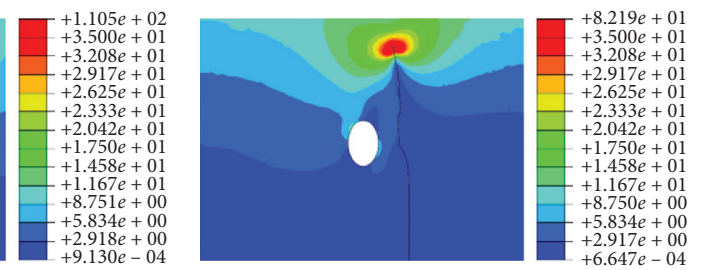

$a=22.7 \mathrm{~mm}$
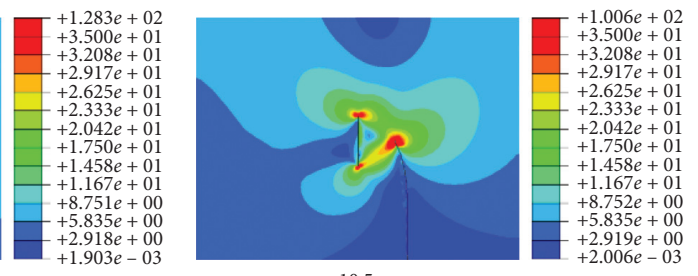

$a=10.5 \mathrm{~mm}$

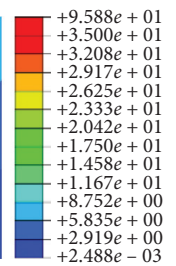

$+2.919 e+00$
$+2.488 e-03$
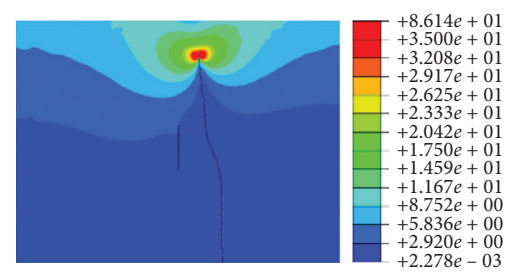

$a=20.9 \mathrm{~mm}$

(b)

FIgURE 12: von Mises stress field with different vertical distance of crack. (a) Specimen $S_{2-1}$. (b) Specimen $S_{4-1}$.

the crack gradually propagates away from the defect and propagates to the direction of the drop hammer loading. Compared with specimen $S_{2-1}$, when the crack deflection angle of specimen $S_{4-1}$ reaches the maximum, the crack does not propagate away from the defect but immediately propagates to the direction of the drop hammer loading. The numerical simulation results show that, with the increase of defect eccentricity, the stress concentration range near the defect decreases, and the effect of defect on crack propagation decreases accordingly.

\section{Conclusions}

In this paper, based on the dynamic caustics experimental method and fractal theory, the dynamic fracture behavior of PMMA specimens with different eccentricity defects under bias precrack conditions is studied. The main conclusions are as follows:

(1) After the drop hammer loading, the evolution of crack propagation behavior can be divided into four stages: crack initiation stage, attraction stage, repulsion stage, and specimen fracture stage. The change of defect eccentricity has no obvious effect on the crack propagation behavior in the crack initiation stage and specimen fracture stage but has a significant effect on the attraction stage and repulsion stage.

(2) With the increment of defect eccentricity of four specimens, the fractal dimension of the crack trajectory near the defect decreases from 1.3100 to 1.2546. The increase of defect eccentricity leads to the improvement of the regularity of crack path. The maximum deflection angle of the crack toward the defect decreases from $29.4^{\circ}$ to $16.7^{\circ}$. The maximum deflection angle of the crack away from the defect decreases from $10.6^{\circ}(e=0)$ to $2.7^{\circ}(e=0.74)$, and as the eccentricity continues to increase, the repulsive effect of defects on cracks decreases significantly.

(3) In the process of crack propagation, affected by the defect, the crack deflects toward the defect. The stress redistributes at the crack tip and $K_{\mathrm{I}}^{d}$ decreases at first and then increases. The valleys of $K_{\mathrm{I}}^{d}$ 
of the four specimens decreased by $0.51,0.37,0.27$, and $0.15 \mathrm{MN} / \mathrm{m}^{3 / 2}$, respectively. The decrement of $K_{\mathrm{I}}^{d}$ decreases with the increase of defect eccentricity. When the defect eccentricity is small, $K_{\mathrm{II}}^{d}$ changes from negative to positive. With the increase of defect eccentricity, $K_{\mathrm{II}}^{d}$ remains negative, and the influence of defect on running cracks weakens.

(4) In this paper, the XFEM is utilized to simulate the effect of defect eccentricity on crack propagation behavior. The stress distribution of specimens with different defect eccentricity is obtained, which explains the cause of crack deflection. The simulation results are in good agreement with the experimental results.

\section{Data Availability}

The data used to support the findings of this study are included within the article.

\section{Conflicts of Interest}

The authors declare that there are no conflicts of interest.

\section{Acknowledgments}

This research was supported by the State Key Development Program for Basic Research of China (Grant no. 2016YFC0600903) and the National Natural Science Foundation of China (Grant no. 51404273).

\section{References}

[1] M. F. Kanninen, C. A. Popelar, and H. Saunders, Advanced Fracture Mechanics, Oxford University Press, Oxford, UK, 1985.

[2] R. Yang, C. Chen, X. U. Wang et al., "Experimental investigation on the influence of different diameter empty holes on the crack growth behavior of blasting," Journal of China Coal Society, vol. 42, no. 10, pp. 2498-2503, 2017.

[3] M. Li, Z. Zhu, R. Liu, L. Zhou, and Y. Dong, "Study of the effect of empty holes on propagating cracks under blasting loads," International Journal of Rock Mechanics and Mining Sciences, vol. 103, pp. 186-194, 2018.

[4] R. Liu, C. Ding, and L. Yang, "Model experiment on dynamic behavior of jointed rock mass under blasting at high-stress conditions," Tunnelling and Underground Space Technology, vol. 74, pp. 145-152, 2018.

[5] H. K. Chen, N. K. Samadhiya, and M. Singh, "Blast induced rock mass damage around tunnels," Tunnelling and Underground Space Technology, vol. 71, pp. 149-158, 2018.

[6] L. SinghGoel, L. Shu-ran, and L. Yan, "Dynamic damage constitutive model of rock mass by comprehensively considering macroscopic and mesoscopic flaws," Explosion and Shock Waves, vol. 35, no. 3, pp. 428-436, 2015.

[7] R. S. Liu, C. X. Ding, L. Y. Yang, and C. Chen, "Hole defects affect the dynamic fracture behavior of nearby running cracks," Shock and Vibration, vol. 2018, Article ID 5894356, 8 pages, 2018 .

[8] H. Xu, M. F. K. Shahriar, and P. Moarefvand, "Experimental and numerical study of crack propagation and coalescence in pre-cracked rock-like disks," International Journal of Rock Mechanics and Mining Sciences, vol. 67, pp. 20-28, 2014.

[9] T. Rabczuk and T. Belytschko, "A three-dimensional large deformation mesh free method for arbitrary evolving cracks," Computer Methods in Applied Mechanics and Engineering, vol. 196, no. 29-30, pp. 2777-2799, 2007.

[10] T. Rabczuk and T. Belytschko, "Cracking particles: a simplified mesh free method for arbitrary evolving cracks," International Journal for Numerical Methods in Engineering, vol. 61 , no. 13, pp. 2316-2343, 2010.

[11] H. Ren, T. X. Zhuang, and T. Rabczuk, "Dual-horizon peridynamics: a stable solution to varying horizons," Computer Methods in Applied Mechanics and Engineering, vol. 318, pp. 762-782, 2017.

[12] Y. Zhong-wen, G. Yang, P. Xu et al., "Analysis of empty hole effect in directional fracture controlled blasting," Explosion and Shock Waves, vol. 35, no. 3, pp. 304-311, 2015.

[13] L. Yang, R. Yang, and P. Xu, "Caustic method combined with laser \& digital high-speed camera and its application," Journal of China University of Mining \& Technology, vol. 42, no. 2, pp. 188-194, 2013.

[14] X Yao, X. Chun-yang, and J. Fang, "Study of dynamic fracture behaviour on three-point-bend beam with off-center edgecrack," Acta Mechanica Sinica, vol. 28, no. 6, pp. 661-669, 1996.

[15] P. Qiu, Z. Yue, and R. Yang, "Mode I stress intensity factors measurements in PMMA by caustics method: a comparison between low and high loading rate conditions," Polymer Testing, vol. 76, 2019.

[16] L. Qing, X. Yao-dong, Q. Yu et al., "Experimental study on impact fracture of cantilever beam-column specimen with prefabricated crack," Journal of Mining Science and Technology, vol. 3, no. 2, pp. 139-147, 2018.

[17] Y. Ren-shu, C. Cheng, F. U. Xiao et al., "Tests for interaction of unilateral offset cracks," Journal of Vibration and Shock, vol. 38, no. 17, pp. 121-127, 2019.

[18] H. Xie, Fractal Applications in the Mathematical Foundations and Methods, Science Press, Beijing, China, 1997.

[19] H. Xie, Introduction to Fractal Rock Mechanics, Science Press, Beijing, China, 1996.

[20] R. Peng, X. He-ping, and J. U. Yang, "Computation method of fractal dimension for 2-D digital image," Journal of China University of Mining \& Technology, no. 1, pp. 22-27, 2004.

[21] L. Mao, X. Lian, and L. Hao, "The fractal calculation of 3Dcracks based on digital volumetric images and its application," Journal of China University of Mining \& Technology, vol. 43, no. 6, pp. 1134-1139, 2014.

[22] Shangqu and S. Sun, "Structural planes surveying and fractal dimension characteristics of tunnel face based on digital photogrammetry," Arabian Journal of Geosciences, 2018.

[23] Y. Ju, C. Xi, Y. Zhang et al., "Laboratory in situ ct observation of the evolution of 3D fracture networks in coal subjected to confining pressures and axial compressive loads: a novel approachc," Rock Mechanics and Rock Engineering, vol. 51, 2018.

[24] A. Lagarde, Static And Dynamic Photoelasticity And Caustics Recent Developments, Springer, Vienna, Austria, 1987.

[25] J. Song, Y. Wang, G. Xiangtao et al., "The mechanism of directional fracture controlled blasting and its application," Journal of Mining Science and Technology, vol. 1, no. 1, pp. 16-28, 2016. 